Voix et Images

voixetimages

\title{
L’esprit du « Lux » ou celui de « Tzëvedzïhr »?
}

\section{Pierre Hébert}

Volume 15, numéro 2 (44), hiver 1990

Pratiques illicites

URI : https://id.erudit.org/iderudit/200845ar

DOI : https://doi.org/10.7202/200845ar

Aller au sommaire du numéro

\section{Éditeur(s)}

Université du Québec à Montréal

\section{ISSN}

0318-9201 (imprimé)

1705-933X (numérique)

Découvrir la revue

\section{Citer cet article}

Hébert, P. (1990). L'esprit du « Lux » ou celui de « Tzëvedzïhr »? Voix et Images, 15(2), 294-298. https://doi.org/10.7202/200845ar d'utilisation que vous pouvez consulter en ligne.

https://apropos.erudit.org/fr/usagers/politique-dutilisation/ 


\section{Roman}

\section{L'esprit du «Lux» ou celui de «Tzëvedzïhr»?}

\section{par Pierre Hébert, Université de Toronto}

Dans le Banquet, Socrate dit qu'il appartient au même homme de savoir traiter également la tragédie et la comédie; on est d'abord poète, c'est-à-dire créateur, le genre étant lui-même secondaire. Il n'en fut pas toujours ainsi des lettres québécoises, où l'esprit de sérieux a longtemps dominé; il faut dire qu'un peuple élu de Dieu et investi d'une mission providentielle n'avait pas les loisirs de se moquer de lui-même. Ce peuple imbu d'un sentiment de perfection ne pouvait qu'ignorer l'existence du relatif, terre de l'humour.

Heureusement, la production romanesque contemporaine donne raison à Platon, témoin les deux romans présentés ici qui occupent sans confusion possible de genre le territoire du sérieux et celui de l'humour. Il s'agit respectivement de Lux $^{1}$, de Pierre Filion, et de Jamädhlavie ${ }^{2}$, de Guy Ménard.

Quatre femmes, dans un bar justement appelé "Lux", dissèquent en paroles leur victime mâle, Bob, dans une lave de mots destructeurs; ces personnages, face au destin individuel, tiennent un discours sans cesse grave. D'autre part, à Tzëvedzïhr - le lecteur malin aura déjà reconnu l'inneffable " t'sé veux dire", lieu principal où se déroule l'action de Jamädhlavie -, le personnage principal Axel revient sur un destin collectif déjà fixé par l'histoire en l'enrobant d'humour.

L'esprit du "Lux", ou: Trois mousquetaires pour notre temps

D'abord, ce début du roman:

Nous l'avions rencontré au Lux, rue Saint-Laurent. Il s'y promenait par ennui, pour voir et être vu dans son plus simple apparat d'humilité. Vêtu d'un ensemble safari un peu trop à la mode, il avait l'air d'un professeur de banlieue qui cherche sa petite scur. Comme les places étaient rares, il a demandé à s'asseoir à notre table; nous avons parlé, il n'était pas pressé [...]. (p. 7)

Tbut le reste de ce roman tient dans cette première rencontre, tant en ce qui concerne les personnages que "l'intrigue». Qui est-il, d'abord, ce professeur de banlieue? Il s'appelle Bob, et il est en congé de maladie, à cause d'un problème de surdité aux origines mystérieuses. Et ce "nous" initial, qui assume une narration 
dont Flaubert eût été fier? Voilà qui est un peu plus compliqué. Il s'agit de trois femmes, Zaza, Haline et M., auxquelles s'adjoindra un peu plus tard une quatrième, Francine. Enfin, lintrigue: Nous avons parlé, et parlé, et parlé... Mais de quoi ?

Car le pauvre Bob, en acceptant de s'asseoir à cette table du "Lux", ignore dans quel filet il vient de se prendre. Pendant un an, dans ce bar ou ailleurs, il sera implacablement remis en question par ces mousquetaires, prêtresses $d u$ new age (p. 228), qui n'auront de cesse de lui enseigner ce qu'elles appellent la science de la vie. Leur jeu est simple: aller chercher les puces dans la tête de tout le monde (p. 36).

Ce qu'il s'en dira des choses dans ce lieu! Chaque rencontre provoquera un combat de coq dont Bob sortira inéluctablement perdant, ces femmes ayant accédé à une philosophie qui dissout complètement les petites rationalisations du prof de banlieue.

Ces trois mousquetaires (qui, bien sûr, sont quatre) vivent pourtant selon des règles simples: Nous n'avons rien de plus extraordinaire que les autres. Seulement trois règles: pas de pensées, pas d'ego, pas d'illusions. (p. 266) Quelque négatives qu'elles puissent paraitre, ces règles de vie ne donnent pas moins à ces femmes une force peu commune, celle d'accéder à la vraie parole, transparente et sans arrière-pensée. Cette parole qui sourd du centre vital de l'être, et non seulement de son cerveau, ne sera possible pour Bob que si lui aussi trouve véritablement sa place dans l'univers en assumant totalement les contradictions de sa condition humaine. Il entrera alors dans un pays nouveau, où les bonnes idées n'existent pas. Tant qu'on fait l'appréciation de nos idées, poursuit Francine, on est fait à l'os. Une idée, c'est ni bon ni mauvais, ça conduit un mouvement qu'il faut comprendre, et rien d'autre (p. 109). Francine n'est pas tant responsable de ses idées que de l'écoute et de la disponibilité qu'elle leur doit.

Prenons la surdité de Bob, par exemple: le groupe des quatre s'évertuera à lui faire comprendre que ce n'est pas, tout simplement, une maladie. Il s'agit au contraire de l'expression physiologique d'un mal d'être où n'arrivent pas à s'équilibrer parole et silence:

Comment pouvait-il savoir que son mal d'oreille était aussi un des fruits de l'ambivalence du silence chez lui, qui participait de ce projet de paix intérieure qu'il était possible pour lui-même et pour nous de croire réel, et aussi l'angoisse de vivre sa propre parole, de supporter son discours, ce qui, certains jours, lui collait les lèvres ensemble, selon ce que Zaza nous confiait au téléphone. (p. 92)

Cette ambivalence s'exprime très bien dans l'attitude de Bob vis-à-vis de son journal intime. Car, n'est-ce pas, à la manière de dizaines de personnages dans le roman des années quatre-vingt, Bob tient un journal qui lui permet de revenir sur les événements qui ont fait l'objet d'une narration antérieure, manière élégante de réinterpréter ce qui s'est passé préalablement. Le sens fragmenté réussit à circuler, péniblement, entre l'interprétation de la narratrice du récit, et le journal de Bob. Mais ce journal lui-même se ressent de cette tension entre la parole et le silence. 
Et qui, au juste, raconte ce récit? De ces quatre femmes, Zaza, Francine, Haline et $M$., la dernière parle peu, et semble toujours prendre des notes. Ce sera en effet elle qui narrera cette série de rencontres avec Bob en recourant au nous, car il y a tellement de choses à dire sur les autres (p. 270). Seulement au dernier chapitre, à la faveur d'un recul vis-à-vis de cette histoire, prendra-t-elle la parole au je, nous livrant les circonstances entourant l'histoire que nous venons de lire.

Lux est, dans un sens, un roman de combat. Peut-être trouvera-t-on la joute un peu trop verbale; mais tous ces "propos et parlures" sont bien ceux de notre temps. L'ère du soupçon est révolue: il est désormais certain que les idéologies sont trompeuses mais que, surtout, la vérité prend pour seul étalon la transparence de l'être qui la proferre. Voici, donc, un roman sérieux, très sérieux même; mais cet esprit n'est pas fondé sur le sentiment d'avoir raison, ou sur une vision absolutiste du monde. Tout au contraire, le sérieux est en l'occurrence inséparable d'une inquiétude qui doit être assumée jusqu'à cette limite où un nouvel humanisme devient possible. Lux essaie d'en tracer les contours...

\section{L'esprit de Tzëvedzïhr: le Québec en spectacle}

Je n'ai jamais eu l'occasion de lire un roman qui ressemble à Jamädhlavie, de Guy Ménard. L'œuvre est tout à la fois un pastiche du style romanesque du XVIII ${ }^{\mathrm{e}}$ siècle, une réflexion sur l'histoire et les mœurs québécoises et, enfin, un petit chef-d'œuvre d'humour.

Jamädhlavie, sur le plan de la structure narrative, s'articule à la manière connue du manuscrit retrouvé par un intermédiaire qui se fait un devoir de le livrer à ses lecteurs, vu son grand intérêt. Ce manuscrit, daté de la toute fin du XVIII e siècle, est adressé à sa nièce par un hobereau, où ce "gentilhomme" raconte ses aventures survenues dans un pays aujourd'hui disparu, la Jamädhlavie. L'oncle en question avait toujours été réticent à raconter son aventure dans cette étrange contrée, et c'est à la faveur de son vieil âge, si l'on peut dire, qu'il se décide à écrire ce qu'on pourrait ici qualifier de "mémoires" et à les léguer à sa nièce. Ce sont ces mémoires, bien sûr, qui constituent l'essentiel du roman.

Axel - c'est le nom du vieil oncle narrateur - choisit un jour de s'enfuir de chez lui, par goût de l'aventure; mais il est enlevé par des brigands, mis aux enchères puis acheté par le fahqir Moustafa. Ce sera là le grand tournant de la vie d'Axel, puisque Moustafa l'initie à son pays, la Jamädhlavie.

Le lecteur du roman de Guy Ménard avait déjà eu la puce à l'oreille lorsqu'il a pu lire cette expression lancée par l'un des ravisseurs dhlaves (oui, dhlaves) d'Axel: "Ahweyk'ris! „Et il aura compris clairement, à peine quelques pages plus loin, que cet étrange pays aujourd'hui disparu, c'est en vérité le Québec.

Les aventures d'Axel au pays dhlave prennent alors un tout autre sens que le lecteur sera constamment appelé à décoder. Bien sûr, dans certains cas, la tâche sera aisée, comme dans le cas de Tzëvedzïhr avec sa montagne Royale, sa Meyhîn qui la sépare en deux parties, et ses problèmes avec l'autre groupe social, les James. Il hésitera peut-être lorsqu'il sera question de l'Outre d'Or et du rêve d'un vizir fou qui y engloutit pendant des années le trésor du royaume (p. 71), mais il y 
reconnaîtra finalement le père du stade olympique. Enfin, le lecteur suivra sans peine Axel lors de cet autre chapitre sur le culte de l'arbre à miel, ou lors de cette initiation à l'histoire dhlave et, en particulier, à l'épisode de l'Union jamadhlave imposée un jour par D'Hiram Pasha.

Après avoir ainsi vécu quelque dix années en pays dhlave, Axel décidera de rentrer chez lui. Mais ce ne sera pas sans avoir tout d'abord vécu un épisode des plus difficiles dans la vie des Dhlaves. En effet, ceux-ci avaient été appelés à se prononcer sur leur destin, à la suite de l'idée de Lëv, qui siégeait sur le Divan de Këvk. Mais c'était sans compter sur la riposte de celui qui régnait à Otwho l, Trüdh Pasha. Il s'ensuivit, paraît-il, après la décision des Dhlaves de ne pas altérer leur union avec les James, une grande déprime où les poètes mêmes sombrèrent dans le silence.

Jamädhlavie vaut d'être lu, pour bien des raisons. Le récit, tout d'abord, propose un voyage à travers l'histoire et la culture québécoises. Certains épisodes sont particulièrement réussis - je pense aux chapitres sur la langue dhlave -, et l'ensemble crée une réflexion et un recul qui permettent une sorte d'autoobservation qui est loin d'être malsaine, surtout lorsqu'elle est assortie d'humour.

Néanmoins, il ne faudrait pas croire que le roman ne pose pas quelques problèmes. Ainsi, dans un certain sens, le texte n'est pas tant une fiction qu'une transcription, brillante certes, mais liée à un référent social ou historique dont la trame peut difficilement émerger, et qu'elle arrive péniblement à remettre en cause ou à critiquer, malgré la distance créée par l'humour. Sans faire de jeux de mots faciles, disons que le discours est ici une friction du réel: il le frotte, le ravive, mais tout en lui demeurant asservi. Il faut dire que, dès l'ouverture du roman, les jeux sont faits: le séjour d'Axel dans le passé dhlave ne pourra ni réécrire, ni relire l'histoire. Pendant que les Fahqirs universitaires - les "vrais", ceux-là - se gargarisent de mots comme "métafiction historique", Jamädhlavie a peine à être davantage qu'une traduction de l'histoire. Ajoutons, en sus, qu'on peut se demander comment ce roman serait lu par quelqu'un qui ignorerait à peu près tout des Québecois: l'histoire d'Axel au pays dhlave perdrait-elle alors tout intérêt?

Et pourtant, il faut le répéter, ce roman manifeste une qualité de style remarquable, compte tenu des contraintes génériques que s'est posées l'auteur. De plus, limagination déborde en ce qui a trait à cette transposition du Québec en pays dhlave, tant au niveau des noms, des évévements que des situations.

Enfin, toute cette entreprise enrobée d'humour vaut d'être notée, ne serait-ce justement que pour la réflexion à laquelle elle nous convie. Dans son Journal d'un inquisiteur, paru en 1960, Gilles Leclerc a fustigé les Québécois pour leur manque d'humour vis-à-vis d'eux mêmes. Jamais un peuple, écrivit-il, n'a tant cultivé l'esprit de sérieux, n'a si peu ri de lui-même à cause, justement, de l'importance du rôle qu'il a cru être le sien dans l'histoire universelle. Le roman de Guy Ménard nous indique sans doute que cet état de choses n'a plus cours aujourd'hui: dans Jamähdlavie, le Québec est vu au moyen de cette soupape de sûreté qui s'appelle le rire, et qui permet de maintenir l'équilibre entre le plus et le moins, entre la vanité et l'auto-dénigrement. Même la "dépression post-référendaire " 
fait désormais partie de l'univers du relatif que sait si bien cultiver l'humour. Certes, là encore, l'humour ne réussit pas toujours à saisir le réel en le transformant; mais en revanche, il permet d'objectiver la condition québécoise en l'amenant au rang de spectacle où ce ne sont ni l'incertitude face à l'avenir, ni le référent historique qui l'emportent, mais plutôt la tension entre les deux. L'histoire est reprise par un discours qui, lui, ne peut jamais s'arracher à la subjugation des faits. Ballotté entre l'aval et l'amont, «Je me souviens* n'avait pas encore connu cette manière de s'exercer.

1 Pierre Filion, Lux, Montréal, Leméac, 1989, 283 p.

2 Guy Ménard, Jamädhlavie, Montréal, Boréal, 1989, 445 p. 